\title{
A CERTAIN DISCONTINUOUS MARKOV PROCESS \\ IN STELLAR DYNAMICS
}

\author{
WERNER TSCHARNUTER \\ Inst. für Theoret. Astronomie, Wien, Austria*
}

\section{Chandrasekhar's Theory}

The basic assumption in Chandrasekhar's approach of statistical stellar dynamics (Chandrasekhar, 1942) is the postulate that a test star within a stellar system being stationary in the sense of collisionless continuum theory suffers random displacements in velocity space generated by the fluctuating part of the gravitational field in a manner that can be described in terms of a random walk. This is equivalent to the assertion that the increments of velocity are regarded as stochastically independent in disjoint time intervals. From this Chandrasekhar derived a diffusion process in velocity space. The equation of motion of the probability density $W(r, u, t)$ in the whole 6-dimensional phase space is then written in the form of a Fokker-Planck-type equation:

$$
\frac{\partial W}{\partial t}+u \cdot \nabla_{r} W+\nabla_{r} \Phi \cdot \nabla_{u} W=\nabla_{u}\left(q \nabla_{u} W+\eta W u\right)
$$

$r, u=$ position, velocity vector; $\Phi=$ gravitational potential of the 'smoothed out' distribution of matter; $q=$ diffusion coefficient; and $\eta=$ coefficient of dynamical friction.

The coefficients $q$ and $\eta$ are related by the condition that a given Maxwellian distribution $f$ of velocities remains invariant for all times, i.e. $(\partial f / \partial t) \equiv \emptyset$.

It is well known that the sample paths of every diffusion process are continuous with probability one. Thus the random velocity of the test star varies continuously in the course of time. Because of this fact the failing of the theory is obvious, since a close encounter of the test star with a field star is able to produce a large change of velocity within a small time interval, clearly contradicting the notion of continuity. Numerical studies on the gravitational n-body problem show that even a velocity higher than the escape velocity can be reached as a result of a single encounter, (Wielen, 1967).

\section{Discontinuous Model}

The aim of the following considerations is to show that Chandrasekhar's basic assumption is more general than the choice of a diffusion process. Thus the phrase 'stochastically independent increments in disjoint time intervals' is not equivalent to the property 'diffusion', since there are infinitely many Markov processes that share only the first property but not the second. If the stochastic variations in velocity space

\footnotetext{
* Now at Universitäts Sternwarte, Göttingen, West Germany.
} 
are approximately describable as a Markov process at all, the question arises whether it is uniquely determined by the properties of the fluctuating part of the gravitational field and whether the jump phenomena mentioned above can be explained by an analysis of its sample paths alone without employing additional assumptions.

To begin with, let us reformulate more definitely the conditions of a random walk. The total increment of velocity within the time interval $(\emptyset, t), t \gg$ characteristic time $T$ during which an elementary fluctuation of the random gravitational field takes place, can be written as a sum of a great number of independent random variables representing the (at least on the average) small displacement in velocity space after the short amount of time $T$ has passed. Now the crucial point is the determination of the distribution law of this sum. This problem, however, is solved exhaustively by the socalled extended central limit theorems of probability theory which were essenially established by Lévy and Khintchin in the 1930's. In general, a convergence to the normal distribution and hence a diffusion is expected, but the probability distribution of the random gravitational field is shown to be asymptotically the Holtsmark-distribution, the characteristic function (Fourier transform) of which is

$$
h(\omega)=\exp \left(-\alpha|\omega|^{3 / 2}\right), \quad \omega=\left(\omega_{1}, \omega_{2}, \omega_{3}\right) .
$$

The Holtsmark-distribution is a stable distribution and belongs therefore to its own domain of attraction (e.g. Feller, 1966). This leads necessarily to a distribution law for the total increment of velocity within $(\emptyset, t)$ with characteristic function

$$
\varphi(\omega)=\exp \left(-\sigma t|\omega|^{3 / 2}\right), \quad \sigma=\alpha \sqrt{T}
$$

$\varphi$ is the Fourier transform of a transition function belonging to a Markov process which is called the stable process with characteristic exponent $\frac{3}{2}$. So far, dynamical friction acting in a purely systematic manner has been ignored. Taking it into account an equation of motion analogous to (1) can be written down:

$$
\frac{\partial W}{\partial t}+u \cdot \nabla_{r} W+\nabla_{r} \Phi \cdot \nabla_{u} W=-\left(-\Delta_{u}\right)^{3 / 4}(\sigma W)+\nabla_{u}(\eta W u)=\mathbf{A}^{*} W(\text { say })
$$

$\sigma$ appears to play a similar rôle as the diffusion coefficient $q$ in (1). The $\frac{3}{4}$-power of the Laplace operator $\Delta_{u}$ is uniquely defined in the sense of its spectral representation. This operator acts on a given function $f$ in the sense that the Fourier transform of $(-\Delta)^{3 / 2} f$ equals to the function $|\omega|^{3 / 2} \hat{f}(\omega), \omega=\left(\omega_{1}, \omega_{2}, \omega_{3}\right)$ where $\hat{f}$ denotes the Fourier transform of $f$. The correct derivation of this term in (4) is not easy and requires sophisticated functional analytic techniques.

From general theorems on Markov processes follows the rightcontinuity of the sample paths. It can also be shown that the mean number of jumps increases to infinity as their heights converge to zero and conversely. This is a very important property because, if one identifies these jumps as the results of far and close encounters respectively, the importance of the far encounters is emphasized on the one hand but spontaneous large changes in velocity due to close encounters are also possible on the 
other hand. These characteristic features are in full agreement with results of numerical experiments on the gravitational $n$-body problem (e.g. Wielen, 1967).

\section{Relaxation Time, Escape Probability}

In the following we shall restrict ourselves to the spatially homogeneous case, i.e. $\nabla_{r} W \equiv \emptyset, \nabla_{r} \Phi \equiv \emptyset$, because it seems impossible to solve Equation (4) in the full 6dimensional space analytically as well as numerically. Unfortunately the assumption of spatial homogeneity is a very serious simplification, because any segregation of mass at the center of a star cluster and the setting up of a halo which are both characteristic phenomena in the dynamical evolution of a stellar system and which will influence its further evolution for their part considerably are entirely ignored. In particular, the escape probability and the number of escaping stars will extensively depend on the space distribution of the field stars.

The following definitions for a relaxation time and an escape probability are even valid, if the stochastic process in velocity space is non-Markovian. Thereafter the relaxation time is defined to be the expected amount of time $\bar{\varrho}$ a test star with initial velocity equal to zero requires to reach the mean velocity of the field stars for the first time. The escape probability is defined to be the probability distribution of the random instant of time $\tau$ the test star gets a velocity greater than the critical escape velocity $v_{\infty}$ for the first time. Clearly, the escape probability is a function of the initial velocity of the test star. By 'stopping' the Markov process at the random 'exit' times $\varrho$ or $\tau$ which are both socalled Markov times for the process all desired quantities can actually be computed.

\section{Numerical Methods and Results}

To the abstract theory as well as to numerical computations the infinitesimal generator A defined by

$$
\mathbf{A} f=-\sigma\left(-\Delta_{u}\right)^{3 / 4} f-\eta u \cdot \nabla_{u} f=\frac{\partial f}{\partial t}
$$

is much more appropriate - by the way, $\mathbf{A}$ has no physical meaning in contrast to its dual $\mathbf{A}^{*}$ ! Both, however, $\mathbf{A}^{*}$ and $\mathbf{A}$ generate the same Markov process. Now it is easy to write down the corresponding equation for the stopped process:

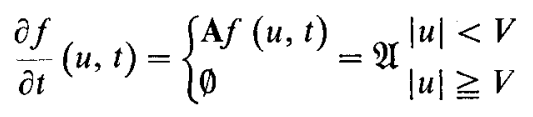

where $V$ denotes either the mean velocity of the field stars or the critical escape velocity. Thus boundary conditions are introduced and the whole theory of Fourier series applies. Infinitesimal and transition operators are then approximated by matrices and functions by vectors.

The coefficients $\sigma$, i.e. $\alpha, T$ in (3), and $\eta$ are given by Chandrasekhar (1942). It is 
easily shown that the Maxwell distribution of velocities is not an invariant distribution of the given Markov process. This fact causes trouble, since the relation between $\sigma$ and $\eta$ cannot directly be established. Numerically it can be defined with the aid of the dual eigenfunction which corresponds to the absolutely least eigenvalue of the operator $\mathfrak{A}$, the velocity $V$ in (6) being chosen sufficiently high. This eigenfunction may be taken as an approximation of the invariant distribution, but the computational difficulties appeared to be enormous. Thereby a reduction of $\sigma$ by a factor 0.85 is suggested having $\eta$ fixed, the problem, however, is far from being solved.

Some relaxation times $T_{R}$ of different star clusters were computed by using the same input parameters as Chandrasekhar (1942) has taken. The quotient $Q=T_{R} / T_{E}$ of corresponding relaxation times were found to depend only on the total number of stars $N\left(Q=2.5\right.$ for $N=50$ monotonically decreasing to $Q=0.07$ for $\left.N=10^{8}\right)$ but almost not on the radii of the clusters.

\section{References}

Chandrasekhar, S.: 1942, Principles of Stellar Dynamics, New York.

Feller, W.: 1966, An Introduction to Probability Theory and Its Applications, Vol. II, New York. Wielen, R.: 1967, Veröff. Astron. Rechen-Inst. Heidelberg, No. 19. 